\title{
The simplest solution
}

Mark Ridley

Reconstructing the Past: Parsimony, Evolution, and Inference. By Elliott Sober. MIT Press: 1989. Pp.265. \$27.50, $£ 25.75$.

How do we know that chimpanzees share a more recent common ancestor with human beings than with amoebas? A less well-known phylogenetic group, such as chimps, humans and gorillas, or wasps, beetles and butterflies, poses the same question in a sharper form. The naive reply, that the answer must come from the fossil record, is of little help. We can already see that a chimp looks more like a human than an amoeba, and for any possible fossil we are again faced with the same question: how do we know that it shares a more recent common ancestor with chimps, or humans or amoebas?

In a general sense, the question is answered by the principle of parsimony. The best estimate of the phylogeny is the one requiring the smallest number of evolutionary changes. Parsimony has more and less exact meanings, and Sober's book is written at a professional level where these different meanings matter; but it is fair to say that all phylogenetic inference depends on some form of parsimony argument, or assumption.

And yet, it is far from clear that evolution is parsimonious. As Joe Felsenstein once remarked, "probably the most parsimonious outcome of evolution would be for it not to occur at all". In morphological evolution, examples of convergence are common, and in some cases the evolution of molecules can - even on the most parsimonious reconstruction be very unparsimonious indeed. The question is not easy.

The various proposed justifications are the subject of Sober's book. Sober is a philosopher who studies biology, and he brings a rare skill to bear upon the philosophical arguments with which biologists (and others) have tried to justify parsimony. He describes the book as an interdisciplinary work, in philosophy, evolution and statistics, but I prefer to divide it into two main parts. Chapters 2 and 3 are philosophical and are concerned with simplicity and induction. Chapters $4-6$ are more biological; they are about evolutionary models and aim to find out under what conditions the principle of parsimony provides a valid inference of phylogeny.

One reaction to the difficulty of justifying parsimony by evolutionary theory has been to deny that its justification is evolutionary at all. It is to be justified instead on philosophical grounds, by

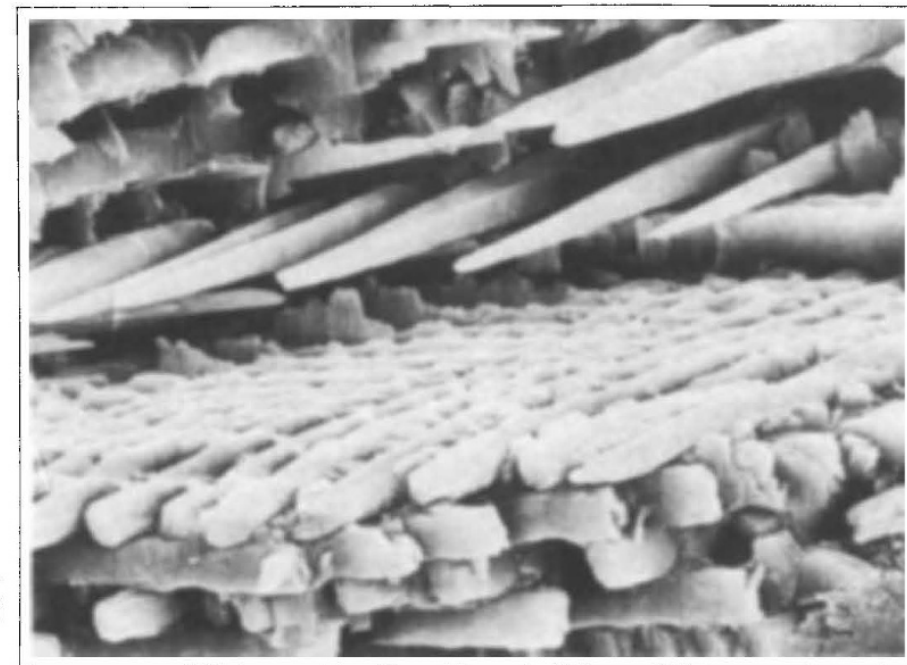

Long in the tooth scanning electron micrograph of rat-incisor enamel, showing two sets of prisms or rods at a 64- $70^{\circ}$ angle to each other and a third array of crystals lying perpendicular to the main rods. Scale, $2 \mathrm{~cm}$ equals $10 \mu \mathrm{m}$. The picture is taken from on Biomineralization newly published by Oxford University Press.

Ockham's Razor or some methodological preference for simplicity. A strange popperian justification has even been put forward, according to which the phylogeny requiring the fewest evolutionary events is best because it is 'least refuted'. Arguments of this kind have particularly appealed to one school of taxonomists, the 'pattern cladists' (who so vexed Nature's editorial writer a few years ago). Pattern cladists like to classify biological species according to their phylogenetic relations, but deny that this practice has anything to do with evolution. The reconstruction of 'phylogeny' (or whatever its non-evolutionary version is called) uses the parsimony principle, and pattern cladists are keen to find a non-evolutionary basis for it.

Sober will have none of this. He accepts that ("perhaps") it is now "the majority view" that scientists prefer simple hypotheses only for reasons of method, but he thinks this is a mistake. A preference for simplicity cannot be justified by methodology, and "an appeal to simplicity is a surrogate for stating an empirical background theory". I would go further. In the absence of evolutionary theory, I deny that a more parsimonious "phylogeny" is any simpler than a less parsimonious one. The parsimonious phylogeny does minimize the number of convergent characters and simplify the hypotheses about convergence. But it does so at the expense of ancestral characters, whose hypothetical numbers it maximizes. If you are trying to reconstruct phylogeny, there is a reason (parsimony) to treat shared characters as ancestral whenever possible; but in the absence of that aim an arrangement that minimizes 'convergence' is no simpler than one that maximizes it. (Convergence and ancestry are meaningless without evolutionary theory; we are just left with a list of characters and character states.)

So a scientific justification for parsimony is needed. Sober considers in detail two opposed arguments, those of Steve Farris and Joe Felsenstein, about the conditions under which the parsimony principle gives the maximum likelihood phylogeny. Felsenstein showed that parsimony works on the assumption that evolutionary change is improbable. I believe this is a good reason for using parsimony, but Sober (and Felsenstein, it seems) is unhappy with it. Sober is mainly interested in establishing that Felsenstein's condition, on the arguments so far presented, is sufficient rather than necessary, and therefore not an assumption of parsimonious inference.

Farris's argument sounds less plausible to begin with. Suppose we have three species (A, B and C) and ten characters suggest the $(A, B) C$ grouping and only one the $A(B, C)$ grouping. Farris remarks that the evidence favours $(A, B) C$ no matter how rare or common evolutionary change is. Even if evolutionary change is common, it is still more likely that at least one of the ten characters is shared from a common ancestor, and not convergent. As the probability of change increases, the one character linking $\mathrm{B}$ and $\mathrm{C}$ is devalued just as much as each of the ten linking $A$ and $\mathrm{B}$. This is true, but the question of reliability remains. The parsimonious inference may still be the best, but it has become less interesting. Felsenstein's condition has more biological appeal: if evolution is improbable, parsimony is reliable.

Many will find these issues excessively esoteric, and Reconstructing the Past is indeed written for fairly expert readers. Biologists (and philosophers, I imagine) with some previous knowledge will get something out of it. But the book is not conclusive; it offers a contribution, rather than a solution, to a research problem. It will, as such, be of most interest to the increasing number of people who are actually working on the statistical inference of phylogeny. Their philosophical appreciation of what they are doing should benefit from Sober's argument. $\square$

Mark Ridley is in the Department of Anthropology and Department of Biology, Emory University, Atlanta, Georgia 30322, USA. 